\title{
Reporte de la experiencia inicial en tomografía de coherencia óptica en enfermedad vascular del injerto
}

\author{
Report of the initial experience in optical coherence tomography for the \\ assessment of cardiac allograft vasculopathy
}

Miguel Angel Larribau, Diego Alfredo Guzzanti, Gustavo Eduardo Irusta, Guido Alfredo D'Amico, Matias Masanet, Federico Welti, Fernando Calderón, Roberto Aníbal Welti

\section{RESUMEN}

Introducción. La enfermedad vascular del injerto es la causa más importante de la falla del órgano trasplantado y muerte después del año. La coronariografía es el gold standard para diagnosticarla, pero presenta baja sensibilidad (10\%).

La tomografía por coherencia óptica (OCT) presenta mayor resolución espacial que el ultrasonido. El objetivo es reportar nuestra experiencia con tomografía de coherencia óptica en la pesquisa de la enfermedad vascular del injerto.

Material y métodos. Pacientes con trasplante cardíaco de más de un año a quienes se les realizó biopsia endomiocárdica, coronariografía y OCT de la arteria descendente anterior. Se diagnosticó enfermedad vascular por angiografía a la presencia de cualquier grado de obstrucción, por OCT al espesor de capa íntima mayor de 0,10 $\mathrm{mm}$ o a intima + media $>0,30 \mathrm{~mm}$.

Resultados. Entre marzo de 2016 y mayo de 2018 se incluyeron nueve pacientes consecutivos, Edad media 45,3 \pm 3 años. No hubo complicaciones vinculadas al procedimiento. La enfermedad vascular del injerto no fue detectada por angiografía, la OCT la detectó en el 55\% de los pacientes según el criterio de espesor de intima $>0,10 \mathrm{~mm}$ y en el $44 \%$ por el criterio intima + media $>0,30 \mathrm{~mm}$.

Conclusiones. La OCT fue segura, la enfermedad vascular del injerto no pudo ser diagnosticada por angiografía mientras que la OCT la detectó en un 55\% de los pacientes. El criterio más sensible para la detección fue el espesor de íntima >0,10 mm.

Palabras claves: tomografía por coherencia óptica, trasplante cardíaco, enfermedad vascular del injerto.

\section{ABSTRACT}

Background. Coronary allograft vasculopathy is an important cause of death and graft failure among patients who had survived more than 1 year after heart transplantation. Coronary angiography remains as the gold standard for diagnosis. The goal of the present study is to report our initial experience with OCT in this population.

Methods. We include consecutive patients who survived 1 year or more after Cardiac Transplantation. Coronary angiography, endomyocardial biopsy and OCT of the LAD were performed. Vasculopathy was considered in any obstruction of the lumen by angiography or Intimal thickness $>0,10 \mathrm{~mm}$ or Intimal + media thickness $>0,30 \mathrm{~mm}$ by OCT.

Results. Nine consecutive male patients were included from march 2016 to May 2018. Age 45,3 \pm 3 years old. No procedural complications were detected.

The angiography didn't find significant obstructions in none of the pts, OCT detected CAV in 5 pts (55\%) by intimal $>0,10 \mathrm{~mm}$ criteria and in 4 (44\%) by Intimal + media $>0,30 \mathrm{~mm}$ criteria.

Conclusions. The OCT study was safe. CAV couldn't be diagnosed by angiography and was detected in 55\% of the patients by OCT. The intimal thickness $>0,10 \mathrm{~mm}$ was the most sensitive criteria for the diagnosis.

Key words: optical coherence tomography, cardiac transplantation, coronary allograft vasculopathy.

Revista Argentina de Cardioangiología Intervencionista 2019;10(2):68-72. https://doi.org/10.30567/RACI/201902/0068-0072

\section{INTRODUCCIÓN}

El trasplante cardíaco está indicado para el tratamiento de la insuficiencia cardíaca refractaria. La enfermedad vascular del injerto (EVI) es la causa más importante de falla del órgano y muerte a tres años del trasplante'. La EVI es una entidad compleja cuya etiología aun no está del todo aclarada.

La hiperplasia intimal es la causa predominante del estrechamiento paulatino y difuso con progresión asintomática hasta estadios avanzados manifestándose por insuficiencia del corazón trasplantado o la muerte, por ello es que cobra vital importancia su diagnóstico precoz. Si bien el seguimiento con coronariografía (CCG) anual es el más utilizado en la práctica, es conocida su baja sensibilidad, razón por la cual el ultrasonido intravascular (IVUS) ha sido incorporado para el diagnóstico precoz de esta entidad.

1. Departamento de Cardiología Hospital Español de Mendoza, Fundación Argentina para el Desarrollo en Salud (FADESA)

$\triangle$ Correspondencia: Dr. Miguel Angel Larribau. Fax: 2614490341. dr.larribau@ gmail.com

Los autores no declaran conflictos de intereses

Recibido: 23/04/2019|Aceptado: 13/05/2019
Las características de la tomografía por coherencia óptica (OCT) en términos de alta resolución para la medición del espesor intimal y la caracterización de la placa ${ }^{2}$ con elevada correlación con la histología la tornan atractiva para la evaluación de la EVI.

\section{OBJETIVO}

El objetivo del presente trabajo es reportar nuestra experiencia inicial con OCT en la detección de la EVI en pacientes con trasplante cardíaco de más de un año de evolución.

\section{MATERIAL Y MÉTODO}

Se incluyeron 9 pacientes que recibieron trasplante cardíaco en Mendoza. Entre marzo de 2016 y mayo de 2018 estos pacientes, consecutivos, fueron sometidos a estudios de protocolo de seguimiento que incluyó la realización de biopsia endomiocárdica, coronariografía y OCT de la arteria descendente anterior en un mismo acto. Para ser incluidos debían tener clearance de creatinina de $60 \mathrm{ml} / \mathrm{min}$ o superior y haber brindado consentimiento informado.

Biopsia endomiocárdica: se realizó según técnica habitual, mediante punción percutánea yugular interna derecha o vena femoral derecha con biótomo Bi Pal 7 Fr (Cordis Car- 


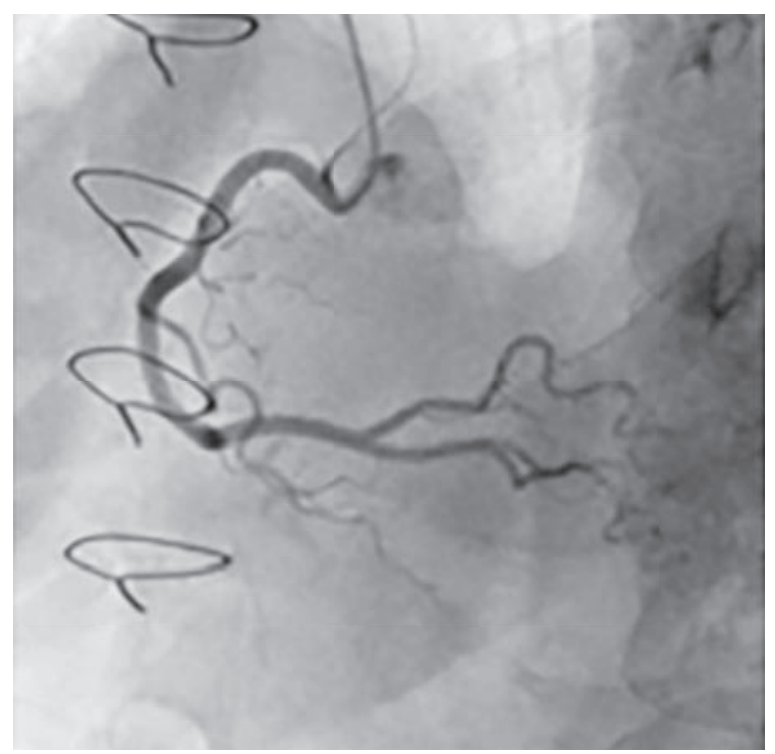

Figura 1. Angiografía de coronaria izquierda. Nótese la ausencia de lesiones visibles y la cuerda guía 0.014" asociada a la sonda de OCT posicionada en el tercio medio de la arteria descendente anterior (flechas).

dinal), obteniendo un total de 5 muestras del lado derecho del septum interventricular, las cuales se remitieron a anatomía patológica en solución de formaldehído.

Coronariografía: se inició al culminar la biopsia, según técnica habitual por abordaje radial o femoral. Se administró heparina no fraccionada para mantener el ACT (activated clotting time) $\geq 300$ s durante el procedimiento. Se comenzó con catéter diagnóstico de coronaria derecha 5 Fr seguido por catéter guía de coronaria izquierda 6 Fr.

OCT: se planeó estudiar de rutina solo la descendente anterior (DA); las otras arterias se examinarían en caso de observar obstrucción en la $\mathrm{CCG}^{2}$. Tras realizar la angiografía se avanzó guía coronaria 0,014 " hasta el tercio distal de la arteria DA sobre la cual se progresó catéter Dragonfly (St. Jude Medical, St. Paul, MN) con el cual se realizó adquisición de imágenes a 100 frames/s en retirada motorizada a $20 \mathrm{~mm} / \mathrm{s}$ desde el tercio distal (después de la segunda diagonal) hasta el tronco de coronaria izquierda. Equipo de OCT C7XR. (St. Jude Medical, St. Paul, MN).

\section{Definiciones:}

- Se considero enfermedad vascular del injerto:

a. Coronariografia: todas las obstrucciones detectables, de acuerdo a las recomendaciones de International Society for Heart and Lung Transplantation ${ }^{3}$.

b. OCT.

i. Espesor intimal $>0,10 \mathrm{~mm}(100 \mu \mathrm{m})$.

ii. Espesor intima + media $>0,3 \mathrm{~mm}(300 \mu \mathrm{m})$.

Se realizó además evaluación cualitativa de las imágenes obtenidas, aplicando las definiciones del consenso internacionals.

Contenido lipídico: aéreas de baja señal, con atenuación, límites poco definidos y sombra detrás de ella.

Fibroso: área de alta señal, homogénea, sin atenuación.

Calcificación: zonas bien delimitadas, heterogéneas, disminución y atenuación de señal.

Se consideró placa vulnerable a la presencia de capa fina del fibroateroma $(<0,065 \mathrm{~mm})$ asociada a core lipídico.

Las imágenes de de la coronariografia y OCT se evaluaron offline por dos de los autores en forma independiente, y en caso de discordancia se tomaría la definición por consenso.

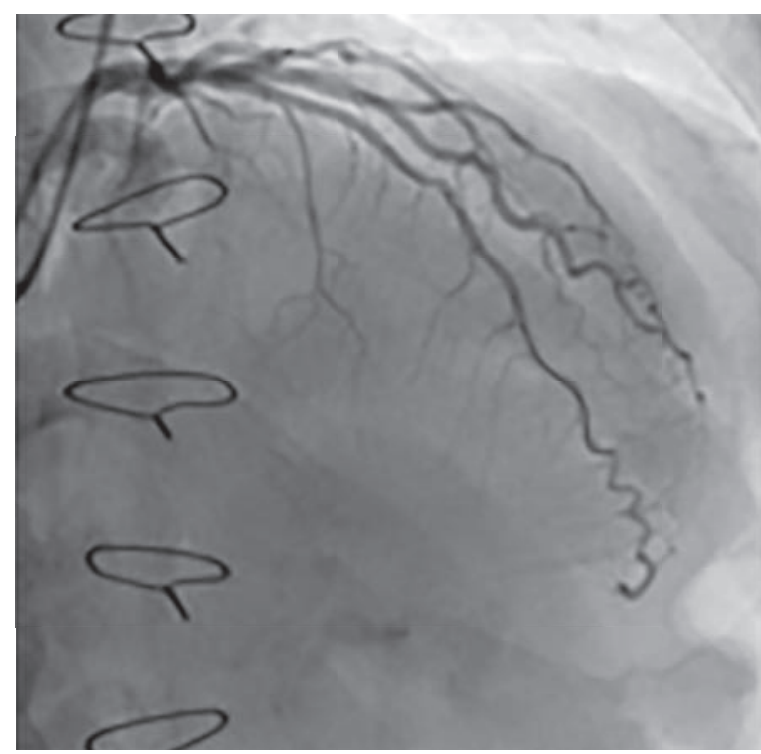

Figura 2. Angiografía de coronaria derecha. Obsérvese la ausencia de lesiones visibles.

\section{RESULTADOS}

Se evaluaron 9 pacientes consecutivos entre marzo de 2016 y mayo de 2018. Todos de sexo masculino, de 45,3 \pm 20 años (rango: 11-73 años), que habían sido sometidos a trasplante cardíaco en Mendoza con 4,1 \pm 3 años de supervivencia desde la cirugía (rango: 1-9 años).

A todos se les realizó biopsia endomiocárdica seguida de CCG y OCT. No hubo complicaciones relativas a los procedimientos y los pacientes fueron externados el mismo día del control.

Todas la imágenes obtenidas pudieron ser analizadas y no hubo discordancia en su interpretación.

La coronariografía no detectó obstrucciones en ningún paciente, la OCT detectó EVI en 5 casos. Los criterios diagnósticos de positividad fueron: 5 por espesor intimal mayor de $0,10 \mathrm{~mm}(55 \%)$ y 4 (44\%) por el criterio íntima + media mayor de $0,30 \mathrm{~mm}$. En tres de ellos (33\%) se detectó placa vulnerable. La biopsia endomiocárdica fue positiva para rechazo celular en un paciente sin EVI.

En las Figuras 1 a 6 se aprecian ejemplos de los hallazgos reportados.

\section{DISCUSIÓN}

El trasplante cardiaco es la terapéutica indicada para la insuficiencia cardíaca refractaria terminal. La sobrevida dentro del primer año se ve amenazada por fallas no específicas del graft, falla multiorgánica, rechazo agudo e infecciones ${ }^{6}$; luego del primer año la enfermedad vascular del injerto es responsable de la mayor tasa de falla del órgano trasplantado y mortalidad ${ }^{3}$. Comienza precozmente después del trasplante y se caracteriza por proliferación intimal progresiva, difusa y concéntrica que compromete los vasos epicárdicos así como arterias intramiocárdicas, arteriolas y capilares. A diferencia de la aterosclerosis, respeta la lámina elástica interna ${ }^{7,8}$. En la Tabla 1 se pueden observar las diferencias entre la EVI y la aterosclerosis.

Inicialmente fue descripta en los estudios de anatomía patológica como una vasculitis muy agresiva. Conforme me- 


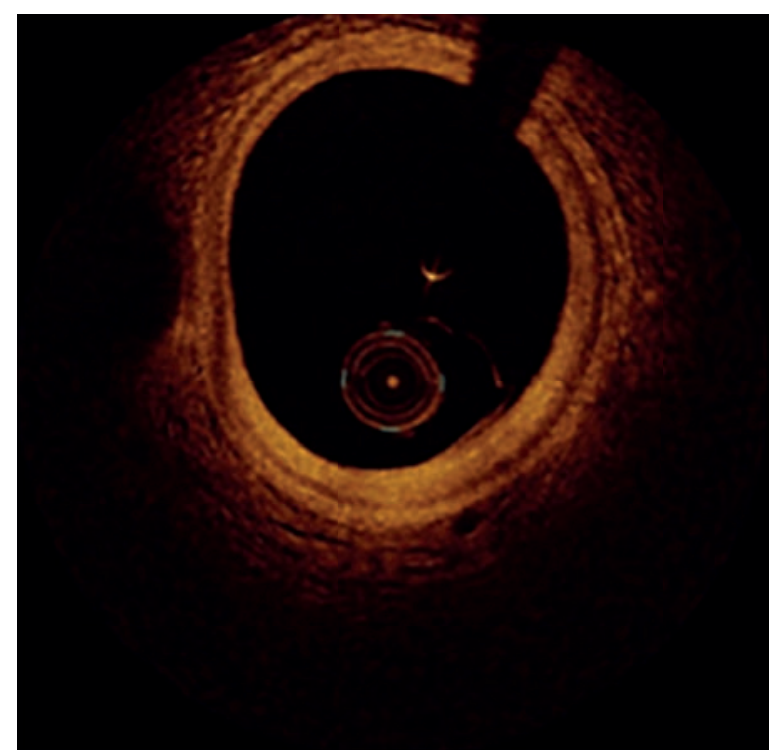

Figura 3. Imagen de OCT. Arriba: corte transversal. Se observan las características de las tres capas normales de la arteria descendente anterior en un paciente sin enfermedad vascular del injerto. Abajo: reconstrucción longitudinal de la arteria.

joró la terapia inmunosupresora y la sobrevida postrasplante aumentó, se la observó en las coronariografías de control. La incidencia reportada es de $29 \%$ y $47 \%$ a 5 y 10 años, respectivamente?

Su fisiopatología es compleja e involucra factores inmunológicos y no inmunológicos que causan inflamación localizada con injuria vascular persistente y daño endotelial. Estudios seriados con IVUS demostraron que la mayor parte del engrosamiento intimal ocurre dentro del primer año. La pérdida de luz obedece fundamentalmente a la hiperplasia ${ }^{10} \mathrm{y}$ en menor medida a remodelamiento del vaso, el cual inicialmente es positivo y luego negativo secundario a constricción de la membrana elástica externa.

La velocidad con la que se desarrolla la EVI y el tiempo de evolución del trasplante son los principales determinantes pronósticos. Puede presentarse precozmente, progresar rápidamente en un año desde una obstrucción leve a severa (>70\%) - denominada fulminante- o aparecer tardíamente luego de los 2 años del trasplante. Esta última en general es de mejor pronóstico que las dos primeras ${ }^{11}$.

Recientemente, un centro canadiense de alto volumen analizó las características de la EVI a lo largo de 30 años, objetivando un cambio en su patrón evolutivo en términos de progresión más lenta y mejor pronóstico. Los autores interpretan que receptores no tan jóvenes, el uso de estatinas y mejores esquemas de inmunosupresión podrían explicar sus hallazgos (encontraron como predictores independientes de progresión de EVI: jóvenes y ausencia de estatinas ${ }^{12}$.

Debido a que carece de síntomas típicos y no puede ser detectada por tests de laboratorio es que resulta de vital importancia el diagnóstico precoz para modificar el tratamiento antes que se establezca la falla del órgano o la muerte del paciente. Por ello la sociedad internacional de trasplante de corazón y pulmón (ISHLT) sugiere la coronariografía anual, siendo este método el gold standard para la detección y seguimiento de la EVI. Así es que los pacientes con EVI severa, definida por obstrucción de tronco $\geq 50 \%$ o dos o más coronarias epicárdicas $\geq 70 \%$, presentan una probabilidad de muerte o retrasplante del $50 \%$ a 5 años $^{3}$. Pero es sabido que

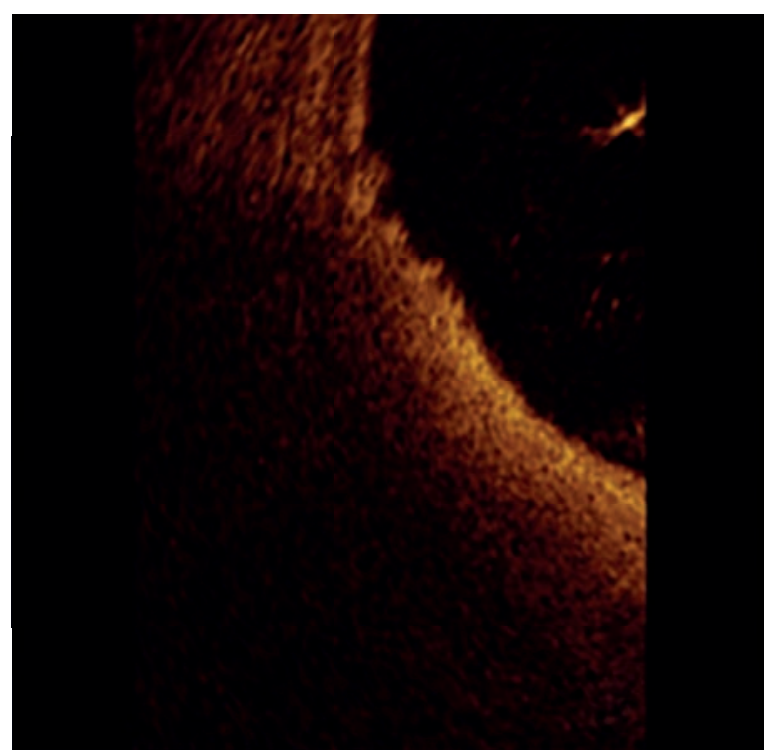

Figura 4. Corte transversal. Nótese el engrosamiento miointimal difuso, concéntrico, fibroso, compatible con EVI. En la intima engrosada entre horas 4 y 8 se aprecian capas de tejido fibroso, elemento predictor de desarrollo de enfermedad angiográfica ${ }^{18}$.

este método posee baja sensibilidad diagnóstica en etapas iniciales ${ }^{13,14}$, por lo que se incorporó el IVUS cuya resolución permite evaluar la pared arterial y cuantificar el grado de hiperplasia. De este modo, un cambio del grosor de íntima + media mayor de $0,3 \mathrm{a} \mathrm{mm} 0,5 \mathrm{~mm}$ en un año define una población de alto riesgo ${ }^{10}$.

Hiperplasia intimal se define por un grosor intimal mayor de $100 \mu \mathrm{m}$. La resolución del IVUS puede detectarla a partir de los $150 \mu \mathrm{m}$, es decir que toda hiperplasia entre $100 \mathrm{y}$ $149 \mu \mathrm{m}$ no podrá ser diagnosticada por IVUS. Debido a que la OCT tiene 10 veces mayor resolución respecto del IVUS y a la buena correlación con la histología para la cuantificación de espesor intimal y caracterización de los componentes de placa aterosclerótica ${ }^{10}$, se está incorporando esta tecnología para el estudio de la EVI.

Hou et al. realizaron IVUS y OCT en una serie de pacientes trasplantados y observaron hiperplasia $>100 \mu \mathrm{m}$ en $66,7 \%$ por OCT y en el 14,3\% por IVUS ${ }^{15}$. Esto podría ser una ventaja de OCT al detectar la hiperplasia intimal por debajo del umbral del IVUS, probablemente en estadios más precoces, con las posibles implicancias terapéuticas que esto conlleva. En un estudio de Liang et al. se correlacionaron los hallazgos de OCT y el rechazo celular; se observó que aquellos sujetos con historia de rechazo de alto grado tuvieron arterias con mayor engrosamiento intimal, con infiltración por macrófagos, que comprometía ramas principales y secundarias ${ }^{4}$. El presente trabajo comunica nuestra experiencia inicial utilizando OCT para evaluar la EVI. Nuestra población estuvo compuesta por pacientes con más de un año de sobrevida al trasplante, entre ellos se encontraba un niño de 11 años de edad. No hubo complicaciones relacionadas con los procedimientos y en todos los casos se obtuvieron imágenes adecuadas para el análisis. No hubo discordancia en la interpretación de las imágenes. En ningún caso se detectaron lesiones coronarias mediante CCG, por ello solo se estudió con OCT la arteria descendente anterior ${ }^{4}$. La detección de EVI varió según el criterio utilizado, a saber: 5 casos por grosor intimal $>0,10 \mathrm{~mm}(55 \%), 4$ de ellos además presentaron grosor íntima + media >0,30 mm (44\%) y 3 de los 5 cumplie- 


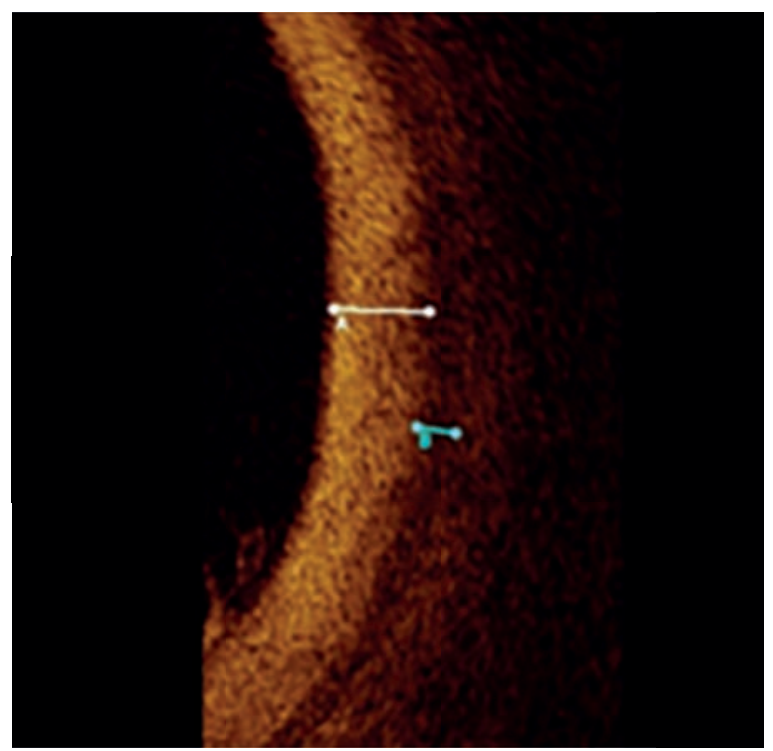

Figura 5. Imagen magnificada. Se puede apreciar el marcado engrosamiento parietal a predominio intimal $(A=0,36 \mathrm{~mm}$ o $360 \mu \mathrm{m})$ de aspecto fibroso homogéneo. Este caso cumple con los dos criterios de engrosamiento (intima e intima + media)

ron criterios de placa vulnerable. Es decir que la sensibilidad fue del $0 \%$ para la coronariografía y del $100 \%$ para la OCT, mientras que el valor predictivo negativo fue alto para OCT y nulo para CCG. La CCG con lesiones severas (grado 3 de la nomenclatura de la ISHLT) tiene alto valor predictivo positivo para eventos, pero la CCG normal no descarta la presencia de EVI. En este grupo de pacientes la OCT positiva tendría alta sensibilidad para su detección precoz y la OCT negativa podría aportar elevado valor predictivo negativo por un año.

La importancia pronóstica del hallazgo podría implicar que los pacientes con hiperplasia podrían sufrir mayor incidencia de eventos adversos (muerte, pérdida del injerto, desarrollo de EVI angiográfica) en los próximos 5 años $^{16}$.

En tres pacientes detectamos placa vulnerable. Park et al. demostraron que la presencia de placa vulnerable diagnosticada al año postrasplante fue predictiva de progresión de $\mathrm{EVI}^{17}$.

Clemmensen et al., en un estudio de OCT, establecieron que la presencia de placas fibrosas en capas y puntos brillantes en la íntima, estos últimos correspondientes a infiltrados de macrófagos, se asociaron a mayor riesgo de progresión no fatal de la EVI, y desarrollaron un modelo predictivo para la misma ${ }^{18}$.

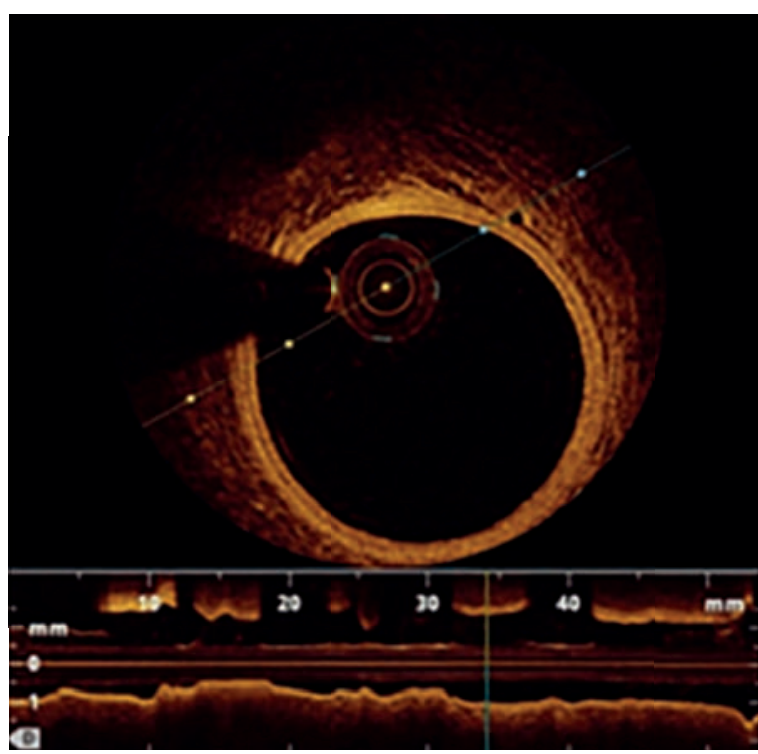

Figura 6. Se puede apreciar el adelgazamiento de la capa intimal por debajo de los $60 \mu m$ y una zona adyacente granular que correspondería a infiltrado de macrófagos, asociado a core lipídico de baja intensidad. Hallazgos compatibles con placa vulnerable.

Habida cuenta del impacto terapéutico de los nuevos esquemas inmunosupresores y que el uso de las estatinas cambió en la evolución de la EVI ${ }^{12}$, es válido plantear la hipótesis que la detección precoz mediante OCT podría modificar la conducta hacia una terapéutica más agresiva, quizás a la medida de cada situación, y monitorear los resultados de la misma con seguimiento con OCT. Por otra parte podría tener utilidad como end point subrogante para estudios futuros.

Por último, debemos puntualizar que el pequeño tamaño de la muestra es la principal limitante del presente reporte, tanto para la evaluación de la concordancia entre los observadores como para los hallazgos obtenidos.

\section{CONCLUSIONES}

En esta pequeña serie de pacientes la OCT fue segura, la enfermedad vascular del injerto no pudo ser diagnosticada por angiografía, mientras que la OCT la detectó en el 55\% de los pacientes. El criterio más sensible para la detección fue el espesor de íntima $>0,10 \mathrm{~mm}$. Serán necesarios futuros estudios para establecer el rol de la OCT para el estudio de la EVI y también su valor como end point subrogante en nuevas investigaciones.

\section{BIBLIOGRAFÍA}

1. Stehlik J, Edwards $L B$, Kucheryavaya AY, et al. The registry of the International Society for Heart and Lung Transplantation: Twenty- seventh official adult heart transplant report-2010. J Heart Lung Transplant 2010;29:1089-103.

2. Kume T, Akasak T, Kawamoto T, et al. Assessment of coronary arterial plaque by optical coherence tomography. Am I Cardiol 2006;97:1172-5.

3. Mehra, MR, Crespo-Leiro, MG, Dipchand A, et,al. International Society for Heart and Lung Transplantation working formulation of a standardized nomenclature for cardiac allograft vasculopathy -2010 Heart Lung Transplant 2010;29:717-27.

4. Dong L; Maehara A; Nazif TM; Pollack AT; et al. Optical Coherence Tomographic Evaluation of Transplant Coronary Artery Vasculopathy With Correlation to Cellular Rejection Circ Cardiovasc Interv. 2014;7:199-206.
5. GuillermoJ. Tearney, Evelyn Regar, Takashi Akasaka, et al.Consensus Standards for Acquisition, Measurement, and Reporting of Intravascular Optical Coherence Tomography Studies. Guillermo J. Tearney, Evelyn Regar. Takashi Akasaka, et al. J Am Coll Cardiol 2012;59:1058-72.

6. Lund $L H$, Edwards $L B$, Kucheryavaya $A Y$, et al. The registry of the International Society for Heart and Lung Transplantation: thirty-first official adult heart transplant report--2014. J Heart Lung Transplant. 2014 Oct;33(10):996-1008. Epub 2014 Aug 14.

7. Tsutsui H, Ziada KM, Schoenhagen P, Iyisoy A, et al. Lumen loss in transplant coronary artery disease is a biphasic process involving early intimal thickening and late constrictive remodeling: results from a 5 -year serial intravascular ultrasound study. Circulation. 2001;104(6):653.

8. Billingham ME. Histopathology of graft coronary disease. J Heart Lung Transplant.1992;11:S38-S44. 
9. Lund LH, Khush KK, Cherikh WS, et al. The Registry of the International Society for Heart and Lung Transplantation: Thirty-fourth Adult Heart Transplantation Report-2017; Focus theme: Allograft ischemic time. J Heart Lung Transplant 2017;36:1037-46.

10. Khandhar SJ, Yamamoto H, Teuteberg JJ, et al. Optical coherence tomography for characterization of cardiac allograft vasculopathy after heart transplantation (OCTCAV study). J Heart Lung Transplant 2013;32:596602.

11. Gao SZ, Hunt SA, Schroeder JS, et al. Early development of accelerated graft coronary artery disease: risk factors and course. J Am Coll Cardiol 1996:28:673-9.

12. Tremblay-Gravel M, Racine N, de Denus S, et al. Changes in Outcomes of Cardiac Allograft Vasculopathy Over 30 Years Following Heart Transplantation. J Am Coll Cardiol HF 2017;5:891-901.

13. Monica CA, Agnihotri A. Cardiac allograft vasculopathy: current knowledge and future direction. Clin Transplant 2011;25:175-84.
14. Cai Q, Rangasetty UC, Barbagelata A, Fujise K, Koerner MM. Cardiac allograft vasculopathy: advances in diagnosis. Cardiol Rev 2011;19:30-5.

15. Hou J, LV H, Jia H, et al. OCT Assessment of allograft vasculopathy in heart transplant recipients. J Am Coll Cardiol Img 2012;5:662-3.

16. Kobashigawa JA, Tobis JM, Starling RC, et al. Multicenter intravascular ultrasound validation study among heart transplant recipients. J Am Coll Cardiol 2005;45:1532-7.

17. Park KH, Sun T, Liu Z, et al. Relationship between markers of plaque vulnerability in optical coherence tomography and atherosclerotic progression in adult patients with heart transplantation. J Heart Lung Transplant 2017;36:185-92.

18. Clemmensen TS, Holm NR, Eiskjær H, et al. Layered Fibrotic Plaques Are the Predominant Component in Cardiac Allograft Vasculopathy Systematic Findings and Risk Stratification by OCT. J Am Coll Cardiol Img 2017;10:773-84. 